\title{
BMJ Open Efficacy of probucol on cognitive function in Alzheimer's disease: study protocol for a double-blind, placebo- controlled, randomised phase II trial (PIA study)
}

\author{
Virginie Lam (10 , ${ }^{1,2}$ Roger Clarnette, ${ }^{3,4}$ Roslyn Francis, ${ }^{4,5}$ Michael Bynevelt, ${ }^{6}$ \\ Gerald Watts, ${ }^{4,7}$ Leon Flicker, ${ }^{8}$ Carolyn F Orr, ${ }^{9}$ Poh Loh, ${ }^{8}$ \\ Nicola Lautenschlager, ${ }^{10,11,12}$ Christopher M Reid, ${ }^{1,2}$ Jonathan K Foster, ${ }^{13,14,15}$ \\ Satvinder S Dhaliwal, ${ }^{16,17,18}$ Suzanne Robinson (D) , ${ }^{2}$ Emily Corti (D) , 1 \\ Mauro Vaccarezza, ${ }^{1,19}$ Ben Horgan, ${ }^{14}$ Ryusuke Takechi (D) ,,2 John Mamo ${ }^{1,2}$
}

To cite: Lam V, Clarnette R, Francis R, et al. Efficacy of probucol on cognitive function in Alzheimer's disease: study protocol for a double-blind, placebocontrolled, randomised phase II trial (PIA study). BMJ Open 2022;12:e058826. doi:10.1136/ bmjopen-2021-058826

- Prepublication history and additional supplemental material for this paper are available online. To view these files, please visit the journal online (http://dx.doi.org/10.1136/ bmjopen-2021-058826).

Received 29 0ctober 2021 Accepted 13 January 2022

Check for updates

(C) Author(s) (or their employer(s)) 2022. Re-use permitted under CC BY-NC. No commercial re-use. See rights and permissions. Published by BMJ.

For numbered affiliations see end of article.

Correspondence to

Professor John Mamo;

j.mamo@curtin.edu.au

\section{ABSTRACT}

Introduction Preclinical, clinical and epidemiological studies support the hypothesis that aberrant systemic metabolism of amyloid beta $(A \beta)$ in the peripheral circulation is causally related to the development of Alzheimer's disease (AD). Specifically, recent studies suggest that increased plasma concentrations of lipoprotein- $A \beta$ compromise the brain microvasculature, resulting in extravasation and retention of the lipoprotein$A \beta$ moiety. The latter results in an inflammatory response and neurodegeneration ensues. Probucol, a historic cholesterol-lowering drug, has been shown in murine models to suppress lipoprotein- $A \beta$ secretion, concomitant with maintaining blood-brain-barrier function, suppressing neurovascular inflammation and supporting cognitive function. This protocol details the probucol in Alzheimer's study, a drug intervention trial investigating if probucol has potential to attenuate cognitive decline, delay brain atrophy and reduce cerebral amyloid burden in patients with mildto-moderate AD.

Methods and analysis The study is a phase II, randomised, placebo-controlled, double-blind single-site clinical trial held in Perth, Australia. The target sample is 314 participants with mild-to-moderate AD. Participants will be recruited and randomised (1:1) to a 104-week intervention consisting of placebo induction for 2 weeks followed by 102 weeks of probucol (Lorelco) or placebo. The primary outcome is changed in cognitive performance determined via the Alzheimer's Disease Assessment Scales-Cognitive Subscale test between baseline and 104 weeks. Secondary outcomes measures will be the change in brain structure and function, cerebral amyloid load, quality of life, and the safety and tolerability of Lorelco, after a 104week intervention.

Ethics and dissemination The study has been approved by the Bellberry Limited Human Research Ethics Committee (approval number: HREC2019-11-1063; Version 4, 6 0ctober 2021). Informed consent will be obtained from participants prior to any study procedures being performed. The investigator group will disseminate
Strengths and limitations of this study

This is the first-in-human prospective randomised placebo-controlled study to assess the efficacy of probucol in delaying cognitive decline in individuals with mild cognitive impairment and mild-tomoderate dementia due to Alzheimer's disease.

- Strengths of the study methodology include randomisation of active/placebo allocation, the doubleblinded nature of the study and the placebo-run in for the first 2 weeks of the study to monitor participant adherence to the treatment schedule.

- The fixed-dose design may be a limitation of the study; further dose-response relationship studies may be indicated.

study findings through peer-reviewed publications, key conferences and local stakeholder events.

Trial registration number Australian New Zealand Clinical Trials Registry (ACTRN12621000726853).

\section{INTRODUCTION}

\section{Background and rationale}

Alzheimer's disease (AD) is a neurodegenerative disorder affecting approximately 50 million people worldwide. Extracellular deposition of amyloid beta $(\mathrm{A} \beta)$ is a hallmark pathological feature of $\mathrm{AD}$ featuring prominently within the hippocampal formation and entorhinal cortex. Amyloidosis is positively associated with cognitive decline in $\mathrm{AD}$ and targeting amyloidosis presently is a therapeutic priority. ${ }^{1}$ Recently, the US Food and Drug Administration Federal Drug Agency approved aducanumab, a treatment which decreases amyloid plaque burden in some patients with $\mathrm{AD} .^{23}$ 
Microvascular disturbances are the first pathological feature of $\mathrm{AD}$ that may include microbleeds; hypoperfusion and or blood-brain barrier dysfunction with changes in the extracellular matrices associated with astrogliosis. ${ }^{45}$ Contemporary treatments for $\mathrm{AD}$ include cholinesterase inhibitors such as galantamine, rivastigmine or donepezil, to support synaptic activity, or memantine to regulate glutamate. ${ }^{6}$ Remarkably, of approximately 400 clinical trials in $\mathrm{AD}$, to our knowledge, none to date have targeted the brain microvasculature, or peripheral metabolism of $A \beta$ to reduce risk for, or progression of $\mathrm{AD}$.

Systemic measures of soluble $A \beta$ positively correlate with cerebral amyloidosis and loss of cognitive capacity in $\mathrm{AD}$ patients. Recent studies demonstrate striking evidence of the high predictive accuracy of plasma $A \beta$ isoforms for individuals who progress to AD-dementia, decades before disease onset. ${ }^{78}$ While a causal association is suggested, the underlying mechanisms by which systemic $A \beta$ metabolism may accelerate the risk of $\mathrm{AD}$ remain unclear. In humans, $\sim 95 \%$ of soluble $\mathrm{A} \beta\left(\mathrm{A} \beta_{1-40}\right.$ and $\mathrm{A} \beta_{1-42}$ isoforms) is associated with plasma lipoproteins, ${ }^{9}$ primarily the triglyceride-rich lipoproteins of hepatically derived very-low-density-lipoproteins and of postprandial chylomicrons. ${ }^{10}$ To directly address the hypothesis of a lipoprotein- $\mathrm{A} \beta$ /capillary axis for $\mathrm{AD}$, mice were engineered to synthesise human $A \beta$ restricted exclusively to the liver to mimic peripheral lipoprotein-A $\beta$ metabolism as seen in humans. Lam et al reported that aberrations in peripheral metabolism of lipoprotein-A $\beta$ result in substantive neurodegenerative changes concomitant with loss of capillary integrity and function, blood-to-brain extravasation of lipoprotein-A $\beta$, marked neurovascular inflammation, and premature hippocampal-learning and memory deficits. ${ }^{11}$ Collectively, these findings provide a strong rationale to consider interventions that target and modulate peripheral metabolism of lipoprotein- $\mathrm{A} \beta$ to mitigate $\mathrm{AD}$ risk.

Probucol is a historic and safe cholesterol lowering drug, clinically used in Japan since 1985, with potent antiinflammatory and antioxidant properties. ${ }^{12-14}$ Probucol was also shown to profoundly attenuate dietary induced synthesis and secretion of lipoprotein-A $\beta^{1516}$ concomitant with cerebral capillary integrity sparing. ${ }^{17}$ In a dietaryinduced diabetic murine model, probucol was also found to support hippocampal-dependent memory recall. ${ }^{18}$ The pleiotropic properties of probucol and significant clinical use experience justifies considering repurposing probucol to test efficacy in supporting cognitive function in patients with $\mathrm{AD}$.

We describe the probucol in Alzheimer's study (PIA study). The study is a phase II placebo-controlled, doubleblind clinical trial assessing the efficacy of probucol in $\mathrm{AD}$. Key outcome measures include cognitive function, regional volumetric changes in brain and cerebral amyloid load.

\section{OBJECTIVES}

The primary objective of this study is to evaluate the efficacy of probucol (Lorelco) on cognitive performance in $\mathrm{AD}$ patients over a 104-week treatment period. The secondary objectives are (1) to evaluate regional volumetric changes and cerebral amyloid abundance in the brain of $\mathrm{AD}$ patients treated with probucol (Lorelco) over a 104-week treatment period, (2) to evaluate improvement or maintenance of quality-of-life parameters in patients with $\mathrm{AD}$ and (3) to assess the safety and tolerability of probucol (Lorelco) in patients with AD.

\section{METHODS AND ANALYSIS}

The methods reporting of this trial follow the recommendations of the Standard Protocol Items: Recommendations for Interventional Trials statement. ${ }^{19}$

\section{Trial design}

This is a single-site, phase II, randomised, double blind, placebo-controlled parallel group study in adults with mild-to-moderate $\mathrm{AD}$. The study will assess the efficacy, safety and tolerability of the treatment of $\mathrm{AD}$ individuals with Lorelco. Participants, study doctors and researchers will be blinded to allocation of the study medication. The maximum study duration is 112 weeks (2 years, 8 weeks), with a treatment period of 104 weeks. There is a 4-week screening phase to ensure all participants have measurable mild or moderate $\mathrm{AD}$ and to ensure eligibility in the study. There is also a 4-week follow-up after the end of the treatment period. We aim to recruit 314 participants for this study. Eligible participants will be randomised in a 1:1 (active:placebo) ratio using permuted block randomisation. Eligible participants will be randomised to a unique participant code, which will be assigned to a participant number. Participant numbers will be randomised to either probucol (Lorelco) or placebo manufactured by Oxford Compounding. The trial coordinator will randomly assign the participant's screening number to a unique participant code at week 1 , day 1 . Participants will be dosed as follows; week 1 and 2: $1 \times$ placebo taken in the morning, with food; week 3: $1 \times 250 \mathrm{mg}$ Lorelco (or matching placebo) taken in the morning, with food; and week 4-104: $1 \times 250 \mathrm{mg}$ Lorelco (or matching placebo) taken in the morning and in the evening, with food. An overview of the study design is shown in figure 1.

\section{Study setting and recruitment}

This study will be based in Australia. All assessments and blood collection will be completed at the Australian Alzheimer's Research Foundation (AARF) based at Hollywood Specialist Centre, Nedlands, Western Australia. Positron emission tomography (PET) imaging will be completed at Sir Charles Gairdner Hospital, Nedlands, Western Australia. MRI will be completed at Envision Medical Imaging, Perth, Western Australia. To reach the targeted sample size, participants will be identified and recruited from the investigators' private and public 

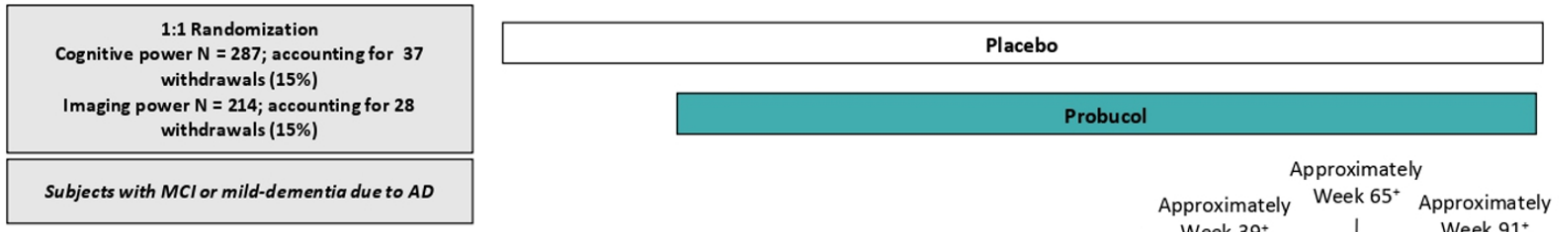

Figure 1 Overview of probucol in Alzheimer's study trial study design. AD, Alzheimer's disease; EoS, end of study; MCI, mild cognitive impairment; PET, positron emission tomography; MRI, magnetic resonance imaging.

patient clinics; referrals from general practitioner or other specialists; phone calls to site via word of mouth; the Alzheimer's Association Research Foundation website; or via database review where participants who have previously given permission for the site to contact them to inform them with new studies. Participants will also be recruited through advertising and editorials on social, print, radio and/or television media; correspondence and promotions via not-for-profit organisations and research partners; website and newsletter content.

\section{Eligibility criteria}

Individuals will be eligible for the study if they meet all of the inclusion criteria and do not satisfy any of the exclusion criteria listed in table 1.

\section{Intervention}

\section{Intervention description}

During the initial recruitment and screening phase, patients will be screened against the exclusion criteria to ensure suitability for participation. During the screening phase, participants will undergo a short cognitive screening assessment, safety assessment, and complete a PET scan to determine cerebral amyloid load. Study medication will commence with a single dose escalation design with all participants receiving initially for 2 weeks $1 \times$ placebo consumed with food, after which baseline measures for cognitive performance, structural and functional brain MRI scans will be completed. Thereafter (week 3), patients receive for 1 week $1 \times$ Lorelco (250 mg) capsule (or matching placebo), taken with food. Commencing week 4, patients receive Lorelco $250 \mathrm{mg}$ two times a day, consumed with food. Study medication will be dispensed at every study visit. Safety evaluations throughout the study will comprise physical/neurological examinations, ECG, vital sign measurements, standard laboratory tests and monitoring for AEs at weeks $3,4,5,15,26,39,52,65,78,91,104$ and 108. At weeks $3,4,6,20,29,47,55,73$ and 81 , the study coordinator will contact the patient or caregiver by phone record to determine any AEs.

\section{Supply of study drug}

Probucol used in this study will be commercially available tablets, Lorelco, produced by Aventis Pharmaceuticals and wholesaled by Otsuka Pharmaceutical Co. Lorelco tablets $(250 \mathrm{mg})$ will be over-encapsulated inside an opaque capsule shell and backfilled with microcrystalline cellulose. Individual doses of Lorelco will be dispensed by the site pharmacy. Matching placebo opaque capsules with no active ingredients and a filler of microcrystalline cellulose will be compounded by Oxford Compounding.

\section{Safety}

Protocol violations should not lead to treatment discontinuation unless they pose a significant risk to participant safety. Trial stopping criteria, dose stopping rules and individual dosage adjustments are indicated in box 1 . 
Table 1 Probucol in Alzheimer's study trial inclusion and exclusion criteria

\section{Inclusion criteria}

1. Must have given written informed consent before any studyrelated activities are carried out and must be able to understand the full nature and purpose of the trial, including possible risks and adverse effects.

2. Adult males and females, aged 18-84 (inclusive) at screening.

3. Diagnosis of $A D$ confirmed by:

- A positive amyloid biomarker (PET scan) indicative of AD pathology.

- Mini-Mental State Examination (MMSE) score of 22 or greater.

- Free and Cued Selective Reminding Test (FCSRT) cueing index of $\leq 0.79$ OR a free recall of $\leq 17$.

- Clinical dementia rating (CDR) global score of 0.5 or 1.0 .

4. Able to take oral medications and willing to record daily adherence to the study drug.

5. QT interval corrected using the Fridericia method (QTCF) $\leq 450 \mathrm{~ms}$ for males and $\leq 460 \mathrm{~ms}$ for females at screening and on day 1 , prior to dose administration.

6. Evidence of adequate hepatic function at screening, as defined by the following:

1. Aspartate aminotransferase (AST) and alanine aminotransferase (ALT) $\leq 2.5 \times$ upper limit of normal (ULN) ( $\leq 5 \times$ ULN if liver metastases are present).

2. Total bilirubin $\leq 1.5 \times$ ULN $(<2.0 \times$ ULN for individuals with liver metastases or documented Gilbert's syndrome).

7. Evidence of adequate renal function, as defined by a calculated creatinine clearance $\geq 50 \mathrm{~mL} / \mathrm{min}$ using the Cockcroft-Gault equation or 24-hour urine collection with plasma and urine creatinine concentrations, respectively.

8. Normal coagulation laboratory assessments at screening.

9. Lipids (total cholesterol, high-density lipoprotein (HDL) and low-density lipoprotein (LDL)) must be within $<1.5 \times$ the upper limit of normal for the local laboratory reference range at the screening visit.

10. Full blood count $(\mathrm{FBC})$ must be within $<1.5 \times$ the upper limit of normal for the local laboratory reference range at the screening visit.

11. Pregnancy:

- Must be of non-childbearing potential (ie, surgically sterilised (hysterectomy, bilateral salpingectomy, bilateral oophorectomy at least 6 weeks before the screening visit)) or postmenopausal (where postmenopausal is defined as no menses for 12 months without an alternative medical cause), or

- If of childbearing potential, must agree not to donate ova, not to attempt to become pregnant and, if engaging in sexual intercourse with a male partner, must agree to the use of acceptable forms of highly effective contraception from the time of signing the consent form until at least 30 days after the last dose of the study drug.

12. Estimated life expectancy of at least 2 years, in the opinion of the investigator.

13. A study partner (partner/spouse/carer) consents to the minimum requirements:

- Will attend at least one screening visit.

- Will be available via phone or in-person to provide information to the study as required.

\section{Exclusion criteria}

1. Recorded number of falls in previous 12 months and during trial. Participants who report multiple falls with potential loss of consciousness.

2. History of QTC-induced prolongation and willingness to limit the use of over-the-counter, or prescription medicines (eg, antihistamines) known to prolong QTc interval. Corrected QT interval using Bazett's formula (QTcB) interval $>450 \mathrm{~ms}$ for males, or $470 \mathrm{~ms}$ for females, as detected by ECG and confirmed by physician. Participants who have a history of QTc-induced prolongation and are unwilling to limit the use of medication.

3. Evidence of abnormal cardiac function as defined by any of the following:

Myocardial infarction within 6 months of cycle 1, day 1.

- Symptomatic congestive heart failure (New York Heart Association>Class II).

- Unstable angina.

- Unstable atrial fibrillation including paroxysmal atrial fibrillation. Medicated, stable atrial fibrillation will be assessed by the study doctor.

- Frequent multifocal ventricular arrhythmia.

4. Unable to swallow oral medications.

5. Gastrointestinal conditions that, in the opinion of the investigator, could affect the absorption of study drug.

6. Use of any prescription or non-prescription (including herbal) medications, or consumption of foods known to be strong QT prolongation within 7 days prior to the first administration of Lorelco and for the duration of the study.

7. Current diagnosis of cancer (within 5 years) and/or undergoing chemotherapy

8. Significant head injury within 5 years.

9. Electrolyte imbalance (eg, on high steroids, pituitary tumours and Addison disease).

10. Hypokalaemia, hypomagnesaemia and hypocalcaemia.

11. Other neurological or psychiatric diagnosis that in the opinion of the investigator could interfere with cognitive function.

12. Major surgery is planned during the conduct of the trial, or a clinical event has occurred in the 6 months preceding study inclusion that may compromise ability to participate for the duration of the study.

13. Evidence of stroke.

14. Current diagnosis with a psychiatric disorder, or taking psychotropic medications.

15. Other excluded medications will be those that are:

- Specifically contraindicated with probucol, based on historic clinical indications for the treatment of cardiovascular disease. Stable use (for at least 3 months) of cholinesterase inhibitors and memantine will be allowed.

- Patients on high dose loop-diuretics or thiazide diuretic medications, will be excluded if taking maximum dose of furosemide or bendroflumethiazid.

16. Self-reported active HIV (HIV-1 or HIV-2), hepatitis B or hepatitis C virus (HCV) at the screening visit.

17. Any inflammatory or chronic pain condition that necessitates regular use of opiates/opioids.

18. Major surgery within 28 days of day 1 week 0 , or minor surgical procedures within 7 days of day 1 week 0 .

19. Hypersensitivity or other clinically significant reaction to the study drug or its inactive ingredients.

20. Participation in another study within the last 4 weeks preceding randomisation and during the present trial.

21. Any other condition or prior therapy that in the opinion of the investigator would make the patient unsuitable for this study, including inability to cooperate fully with the requirements of the study protocol or likelihood of non-compliance with any study requirements.

AD, Alzheimer's disease; PET, positron emission tomography.

\section{Adverse events}

The investigators will report any serious adverse events (AEs) occurring during the clinical trial, independent of direct causal relationship with the treatment, within 24 hours. Unblinding will be permissible in the event information is required to ensure the participants safety in case of an $\mathrm{AE}$. The study doctor will provide Oxford Compounding with the unique participant code to unblind the participant. All AEs will be reviewed by the independent data safety and monitoring committee (DSMB), specifically appointed for the trial.

\section{Participant withdrawal}

If a participant decides to withdraw from the project, participants are asked to notify a member of the research team. If a participant withdraws consent during the 
Box 1 Probucol in Alzheimer's study trial stopping

criteria and intervention dosage adjustments

\section{Trial stopping criteria}

- Substantial deviations from the approved protocol.

- Adverse-effects of unexpected type, severity or frequency are encountered.

- As the trial progresses, the continuation of the trial would disadvantage some of the participants as determined by the investigator group, trial monitors or the data safety and monitoring committee.

*All participants will take part in a safety evaluation 4 weeks following discontinuation.

Dose stopping criteria and individual dosage adjustments

Dosing may be stopped or modified if suspected adverse drug reactions, changes in vital signs, ECGs or clinical laboratory results are observed, and these changes pose a significant health risk. Of particular note for dose discontinuation are the following criteria:

- Corrected QT/QTc interval is in excess of $500 \mathrm{~ms}$, or if it increases $>50 \mathrm{~ms}$ compared with baseline (baseline will be calculated as the mean of two time points, screening and baseline day 1).

- Occurrence of QT/QTC interval prolongation in association with symptoms of arrhythmia.

- Subsequent prescription of a drug with potential QTc prolongation (antimalarial; macrolides; quinolones; triazole antifungals; antiarrhythmic; antiemetic; antidepressant; antipsychotics).

- Evidence of liver dysfunction, or primary biliary cirrhosis.

- If for any reason the investigator team determines that continued participation in the trial is not in the participant's best interest.

research project, the study doctor and relevant project team members will not collect additional personal information from the participant. However, personal information already collected will be retained to ensure that the results of the research project can be measured properly and to comply with law. Participants will be made aware that data collected by the Sponsor up to the time the participant withdraws will form part of the research project results.

\section{Adherence}

The 2-week placebo period will serve to monitor participant adherence to the treatment schedule. Participants will also be required to return the study treatment webster packs at every clinic visit. In the event adherence falls below $80 \%$, participants will be re-trained in the administration of the study medication. If adherence continues to be below $80 \%$ the participant will be withdrawn from the study.

\section{Concomitant care}

This study allows for 'usual clinical care'. Some medications or treatments may not be permitted during participation in this study. The study doctor will collect information about concomitant medication use during every study visit and safety screening. Participants will not be permitted to take part in other studies/investigational treatments for $\mathrm{AD}$ or other health conditions while taking part in this study.

\section{Study procedures}

For the overall schedule of the trial and the exact timing of each procedure, refer to the Schedule of Assessments (table 2).

\section{Screening assessments \\ Informed consent}

The information and informed consent form (ICF) will be provided to patients at screening and signed consent must be provided prior to any study procedures being performed.

\section{Medical history}

A full medical history will be obtained at screening, including a detailed neurological history, other medical and surgical history, medication history and drug allergies. Demographic data including gender, ethnicity and race will be recorded.

\section{Height and weight}

Body height $(\mathrm{cm})$ and weight $(\mathrm{kg})$ will be measured, and body mass index will be calculated.

\section{Pregnancy test}

Female patients (women of childbearing potential only) will complete a urine human chorionic gonadotropin (hCG) pregnancy test at screening, baseline and again at end of study (EoS) visit.

\section{Safety and tolerability assessments}

Safety will be determined by evaluating physical and neurological examinations, vital signs, clinical laboratory parameters, 12-lead ECGs and AEs. Abnormal vital signs assessments, clinical laboratory safety tests, ECGs and physical examinations that are judged by the principle investigator as clinically significant will be recorded as AEs or serious adverse events (SAE). The timing of all safety assessments is presented in table 2 .

\section{Vital signs}

Vital signs assessments will include systolic and diastolic blood pressure, heart rate (HR), respiratory rate (RR) and body temperature. Patients should be resting in a supine position for at least $5 \mathrm{~min}$ prior to and during vital signs measurements.

\section{Clinical laboratory safety tests}

Fasted blood samples (minimum 8 hours fast) will be collected by venepuncture at screening, baseline, weeks $3,4,5,15,26,39,52,65,78,91$ and 104 weeks (an estimated $13 \mathrm{~mL}$ of blood will be collected per visit and a total of $160 \mathrm{~mL}$ will be collected over the 2-year study).

Blood samples for haematology, serum biochemistry (including liver function tests) will be collected at selected time points throughout the study (see table 2). Test results will be monitored for potential AEs, including gastrointestinal bleeding (haemoglobin) and rhabdomyolysis (plasma CK). Apolipoprotein E genotype will also be determined. 


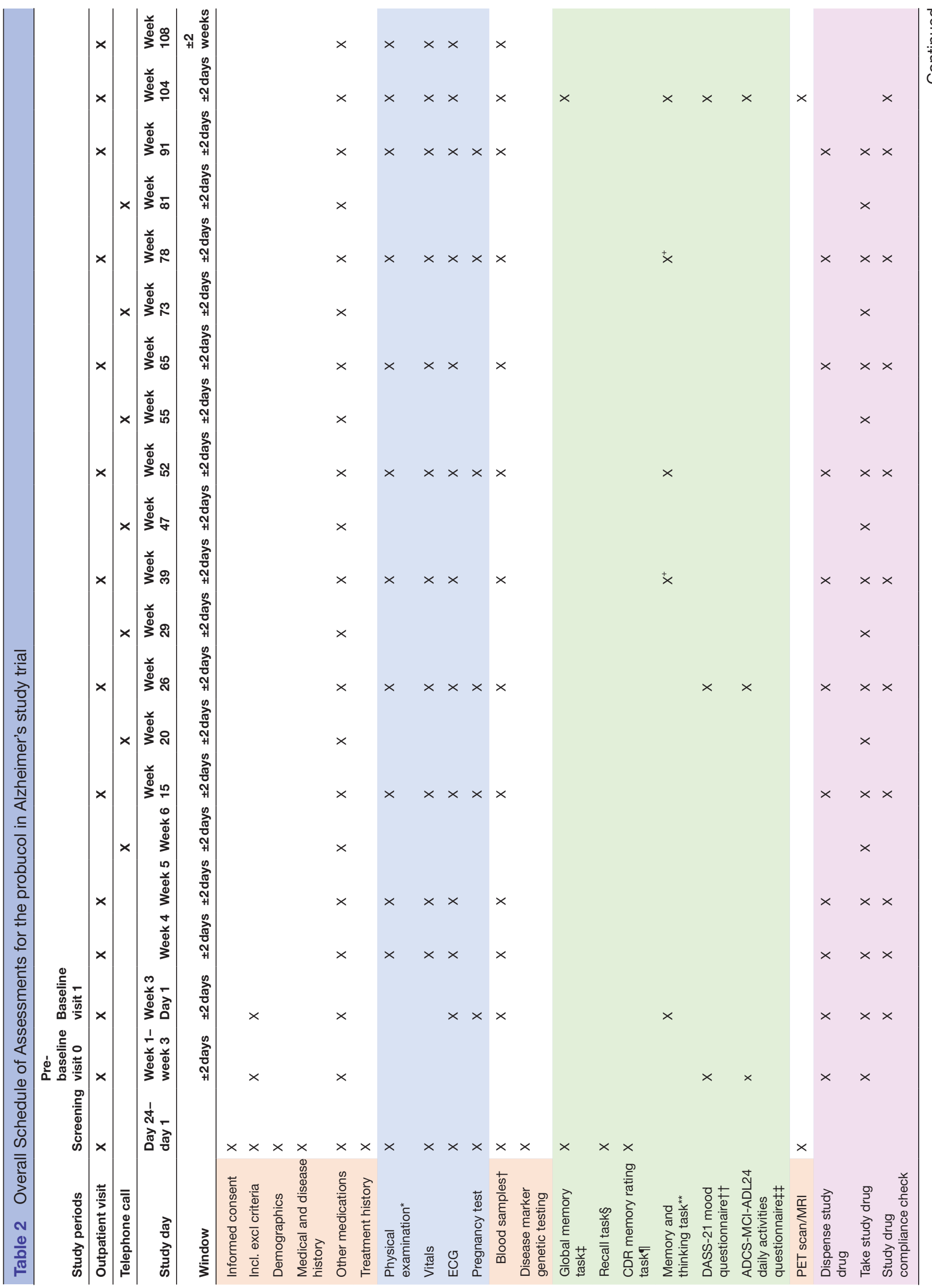




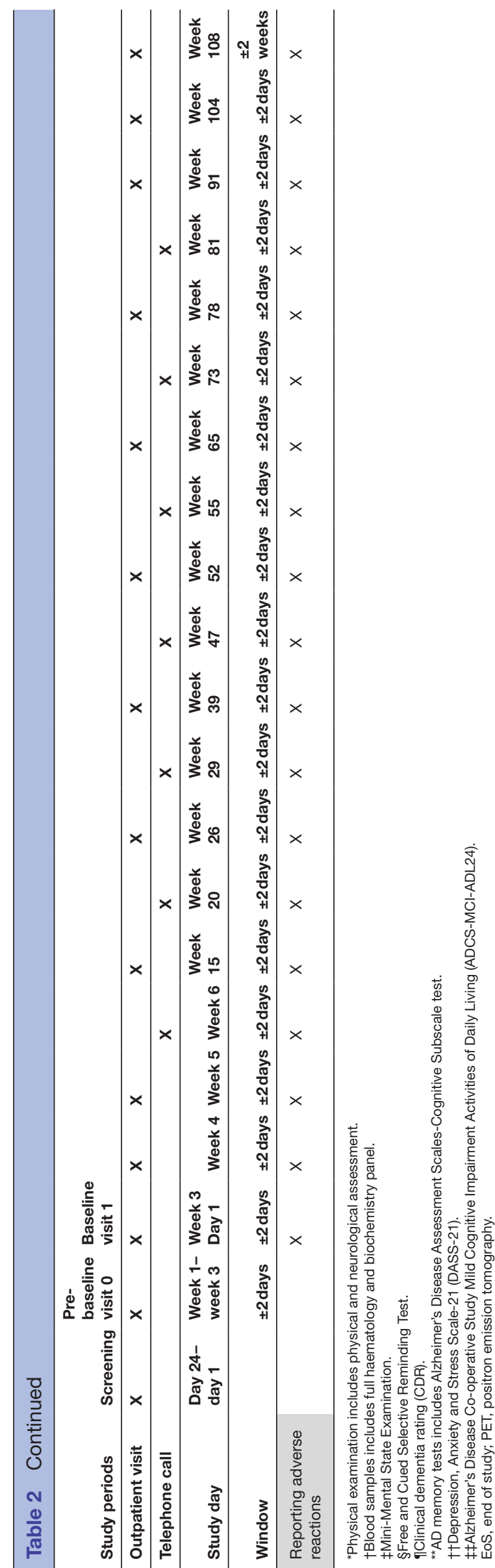

ECG

Twelve-lead ECGs will be assessed (including but not limited to the measurements of ventricular HR, PR interval, RR interval, QRS duration, QT interval and QTCF). Screening and prior to first dose, triplicate 12-lead ECGs (collected within $5 \mathrm{~min}$ with each reading separated by at least $1 \mathrm{~min}$ ) will be taken to establish eligibility at baseline. Triplicate ECGs will also be recorded at the EoS visit. The average value for the triplicate will be used for assessing QTcF inclusion criteria. All other ECGs will be single readings.

ECG normal ranges are as follows: PR interval: 120 ms-220 ms (inclusive); QRS duration: <120 ms; QTcF $\leq 450 \mathrm{~ms}$ (males); QTcF $\leq 460 \mathrm{~ms}$ (females); HR $45-100$ beats/min (inclusive).

Physical examination

A full physical examination will be performed at screening and at the EoS visit. The full physical examination will include, at a minimum, assessment of the following systems: skin, head, ears, eyes, nose and throat, lymph nodes, heart, chest, abdomen and extremities, and a neurological examination (assessment of speech, cranial nerves, peripheral nerves, motor power, deep tendon reflexes, sensation, coordination and gait) and any other focused assessments suggested by the presence of specific symptoms. All other scheduled assessments will be symptom-directed.

\section{Cognitive screening assessments}

Free and Cued Selective Reminding Test

The Free and Cued Selective Reminding Test (FCSRT) ${ }^{20}$ assessment will be performed at screening only for eligibility determination. A cueing index of $\leq 0.79$ is required for study entry. The FCSRT is a cued recall test that uses a controlled encoding technique to ascertain that impairment in recall and cueing are due to a memory deficit rather than a failure of encoding.

\section{Clinical Dementia Rating scale}

The Clinical Dementia Rating $(\mathrm{CDR})^{21}$ provides two scores, a global score (GS) and a sum of boxes (SOB). The GS distinguishes a participant's level of impairment into the following categories: 0 (normal); 0.5 (questionable dementia; 1 (mild dementia); 2 (moderate dementia) and 3 (severe dementia). The SOB is scored from 0 to 18 with higher scores indicating a greater level of impairment. The scale covers six domains: memory, orientation, judgement and problem solving, community affairs, home and hobbies and personal care.

\section{Mini-Mental State Examination}

The Mini-Mental State Examination (MMSE) ${ }^{22}$ is a brief, widely used 30-item assessment of global cognition examining orientation, registration, calculation, recall, attention and language. The spelling of WORLD backwards will not be used in this protocol. Participants who score $<22$ at screening will be ineligible for study entry. MMSE will be assessed at screening and EoS. 
All cognitive assessments will be performed by an independent assessor who will be blinded to treatment allocation.

\section{Outcome measures}

\section{Primary outcome measures}

The primary outcome measure will be the change in the Alzheimer's Disease Assessment Scales-Cognitive Subscale test (ADAS-Cog).$^{23}$ The ADAS-Cog is the most widely used test to measure cognition in RCT's for AD. The ADAS-Cog consists of the following tasks: Word Recall Task; Following Commands; Constructional Praxis; Delayed Word Recall; Naming Objects and Fingers; Ideational Praxis; Orientation; Word Recognition; Spoken Language; Comprehension and Word Finding Difficulty. The ADAS-Cog 12 has a total scoring range of $0-70$, where 70 represents the most severe impairment and 0 represents the least impairment. The full suite of ADAS-Cog will be assessed at baseline, 26, 52, 78 and 104 weeks. At the discretion of the study sponsor, the extended ADAS-Cog, with the additional tasks Maze and Number Cancellation, may also be administered.

\section{Secondary outcomes}

A secondary outcome measure will be assessment of brain morphometry and volume determined via MRI at baseline (preintervention) and EoS. The MRI protocol will include the acquisition of three sets of data (1) Volumetric isotropic $\mathrm{T} 1 \mathrm{scan}(6.5 \mathrm{~min})$. This will allow voxel wise segmentation and volumetric analyses (eg, grey matter volume) to assess volume changes in characteristic locations which can yield diagnostic accuracy on approximately $90 \% .{ }^{24}$ Mesial temporal lobe (hippocampus and entorhinal cortex via Scheltens grading), global cortical atrophy (Pasquier scale) and parietal atrophy (Koedam score) as well as inferior lateral ventricle size will be assessed. Brain volume indices indicate that patients with $\mathrm{AD}$ have accelerated rates of brain volume loss of up to $4.5 \%$ per year compared with normal controls $(1 \%)$. (2) 3D FLAIR scan (3.5 min). This will demonstrate the small vessel ischaemic lesion load which will be scored according to the Fazekas method. ${ }^{25}$ (3) Susceptibility weighted imaging (SWI) - a means of measuring micro bleed load and indicator of amyloid angiopathy and for the purposes of Quantitative Susceptibility Mapping ( 7 min).$^{25}$ This method quantitates regional brain iron content which is altered in $\mathrm{AD}$ compared with normal controls. Mesial temporal, basal ganglia, cingulate, cortical region of interest comparisons will be performed at baseline and at treatment completion. Visible micro bleeds on the SWI will be graded using the Brain Observer Micro-Bleed Scale. ${ }^{26} 27$

Cerebral amyloid load will be assessed as an additional imaging outcome measure. Brain amyloid imaging will be done with amyloid tracer PET scans at baseline and at EoS. Dynamic and static PET imaging will be acquired. Analysis will include (1) visual assessment of amyloid load, (2) quantitative assessment of amyloid burden, including standard uptake value ratio and (3) dynamic imaging for blood perfusion measures.

Quality of life will be assessed as a secondary outcome measure via the Alzheimer's Disease Co-operative Study Mild Cognitive Impairment Activities of Daily Living (ADCS-MCI-ADL24).$^{28}$ The ADCS-MCI-ADL24 is a study partner 24-item questionnaire evaluating perceived difficulties with functioning in several activities of daily living across a variety of domains. The Depression Anxiety Stress Scale (DASS-21), a self-report 21-item will be used to determine levels of depression, anxiety and stress. ${ }^{29}$ Participants are read a statement and asked to rate each statement on a 4-point scale as to how much it relates to them. DASS-21 will be administered at day 1 / week 1 , week 26 and EoS.

\section{Statistics}

\section{Estimated sample size and power}

Estimated sample sizes are calculated for the two outcome measurements: ADAS-Cog and grey matter atrophy (hippocampal). In order to ensure that the study has sufficient power to detect differences in both of the primary outcomes, the sample size chosen is the maximum of that calculated for each primary outcome.

The primary analysis is an intention-to-treat analysis and will include all randomised participants. Data will be analysed using both generalised estimating equations (GEE) and Bayesian analysis. The analysis of primary endpoints will use linear mixed-effects models, with random slopes and intercepts. For the ADAS-Cog, using mixed model analysis published estimates from the Alzheimer's disease neuroimaging initiative (ADNI) cohort ${ }^{30}$ suggest a sample size of $125 \mathrm{AD}$ participants per trial arm (total $\mathrm{N}=250$ ) will be required for power at 0.8 to detect a drug effect of $25 \%$ over 2 years and assuming a decline from baseline of 1.10 standardised units on the composite (SD change $=0.83$ ). For the MRI markers, Ledig et al reported the sample sizes required for a $25 \%$ intervention reduction over 2 years based on 322 patients with $\mathrm{AD}$ (with 117 followed for 24 months) and a reduction of $10.2 \%$ (6.2) for hippocampus. ${ }^{31}$ Sample size calculations based on hippocampal volume suggest that 93 participants per treatment arm are required (total $\mathrm{N}=186$ ). Assuming a $20 \%$ attrition rate, a sample of 314 individuals will be recruited for the cognitive study, and 233 individuals will be randomly chosen for the imaging study.

\section{Statistical analysis}

\section{Outcomes}

The primary analysis is an intention-to-treat analysis of all randomised participants. Data will be analysed using both GEE and Bayesian analysis. The analysis of primary endpoints will use linear mixed-effects models, with random slopes and intercepts. Analysis of all primary and secondary endpoints contrasting probucol and placebo, after adjusting for covariates, will use mixed effectsregression with 'random' intercepts and slopes (as has been used for power calculations). Mean differences and 
associated 95\% CIs will be presented for the 'fixed' effect of probucol treatment. No formal interim analyses are planned at this time.

\section{Additional analyses}

Further analysis investigating the relationship between change scores (postintervention minus preintervention scores) for the primary ADAS-Cog with MRI volumes (total grey matter, hippocampus and medial temporal lobe volumes) and specific blood biomarkers (eg, plasma lipoprotein-A $\beta$ ) will be considered using Pearson (or Spearman where appropriate) correlation analysis. For all other correlations between recorded variables that lack an a priori hypothesis, control of statistical errors will be carried out using Holm-Sidak corrections for multiple comparisons.

If probucol treatment is successful, a directed acyclic graph Bayesian network analysis will be carried out a posteriori on variables identified to be significant predictors of either grey matter arrest or neuropsychological performance to better elucidate mechanisms of the effect of probucol. Greedy equivalence search will be used to identify statistical conditional dependencies between variables and directionality will be estimated using the linear, non-Gaussian, acyclic causal models approach. ${ }^{32}$ Goodness of fit will be estimated using a $\chi^{2}$ test contrasting the identified model against a saturated model.

In addition to Bayesian analyses, the traditional general linear model analysis will also be used to compare probucol to placebo, after adjusting for covariates. The GEE method, which extends the generalised linear model to allow for analysis of repeated measurements or other correlated observations, will also be used. Missing data on the ADAS-Cog will not require data imputation. The scoring methodology for the ADAS-Cog as proposed by Verma et al will be used as it estimates cognitive impairment using the set of items answered by the patients. ${ }^{23}$ Any additional missing data will be identified using missing values analysis and will be replaced using multiple imputation where appropriate. Mean difference and associated $95 \%$ CIs will be presented. Data will be analysed using Stata V.16.

\section{Data and safety monitoring \\ Data management}

Data will be collected by study delegated personnel on paper source maintained in a participant study binder in secure facilities at AARF. Identifiable data will be stored securely and kept in a locked cabinet with access restricted to the investigator team, site and monitoring personnel. All other data will be de-identified to sure confidentiality of participant data. Data will be stored electronically on password protected web-enabled clinical trial data electronic management system (REDCap) located in an ISO27001 compliant facility at Curtin University. Clinical records collected at recruitment will be kept in a locked cabinet in a locked office at the site and will collectively be housed in secure facilities at the AARF. Participants' study information will not be released outside of the study without the written permission of the participant. All data will be securely archived as per the Sponsor's data policy for a minimum of 25 years.

\section{Trial monitoring and formal committees}

The trial monitoring committee, comprised the principal investigator, key trial staff including the trial manager, a nurse representative and a consumer representative, are responsible for trial setup, ongoing management and promotion of the trial. The trial steering committee (TSC), comprising the investigator team including geriatricians, cardiologists, neuroradiologists, nuclear medicine physicians, neuropsychologists, a biostatistician, clinical biochemists, consumer and community representatives, will provide overall supervision of the study and are responsible for interpretation and dissemination of results.

AARF employs independent data auditors who will monitor and audit compliance of data entry/management, legislation, regulations, guidelines and codes of practice, at quarterly intervals. Findings from each audit will be discussed with the study coordinator and thereafter with the investigator team to ensure any action items are addressed promptly and appropriately.

An independent DSMB will oversee the safety aspects of the study. The DSMB consists of members with expertise in clinical pharmacology, biostatistics, clinical trial design and clinical cardiology. Members of the DSMB will not be investigators of the study nor will they have any conflict of interest with the investigators. The committee will meet periodically to advise the TSC on the progress of efficacy and safety data as it accumulates throughout the course of the study. The TSC and DSMB will provide independent oversight of the study.

\section{Patient and public involvement}

The study was developed in consultation with a consumer advocate representing the Consumer and Community Involvement Programme (https://cciprogram.org/). The trial will be overseen by the TSC, including patient and public members.

\section{ETHICS AND DISSEMINATION \\ Ethics approval}

The PIA study has been approved by Bellberry Ltd Human Research Ethics Committee (HREC2019-11-1063; refer to online supplemental file 1 for the approved study protocol). This trial is registered in accord with the WHO Trial Registration Data Set. The Universal Trial Number is U1111-1259-0486. Where applicable, approved protocol amendments will be communicated to the trial personnel and relevant committees.

\section{Informed consent and withdrawal from the study}

Participants will be in the mild stages of dementia and therefore are expected to be able to provide informed consent. An investigator or research staff delegate will explain all study procedures and possible risks to the 
participant. The participant and their nominated study partner will have an opportunity to have all questions answered and thereafter will sign and date the ICF, indicating willingness to participate in the study with an option to consent for blood samples stored and used for future research purposes (online supplemental appendices A-D). Participants will be informed prior to consent that they can withdraw at any time without their care being affected in any way.

\section{Dissemination}

Participants will be updated about the progress and results of the study via presentations or newsletters from the investigator group. The results will be disseminated via peer-reviewed publications, key conferences and local stakeholder events, under the Consolidated Standards of Reporting Trials guidelines. ${ }^{34}$

\section{Trial status}

This study is in the process of recruiting participants and expected to complete in 2026.

\section{Author affiliations}

${ }^{1}$ Curtin Health Innovation Research Institute, Faculty of Health Sciences, Curtin University, Perth, Western Australia, Australia

${ }^{2}$ School of Population Health, Faculty of Health Sciences, Curtin University, Perth, Western Australia, Australia

${ }^{3}$ Australian Alzheimer's Research Foundation, University of Western Australia,

Nedlands, Western Australia, Australia

${ }^{4}$ School of Medicine, University of Western Australia, Crawley, Western Australia, Australia

${ }^{5}$ Department of Nuclear Medicine, Sir Charles Gairdner Hospital, Nedlands, Western Australia, Australia

${ }^{6}$ Neurological Intervention and Imaging Service of Western Australia, Sir Charles Gairdner Hospital, Nedlands, Western Australia, Australia

${ }^{7}$ Cardiometabolic Service, Department of Cardiology and Internal Medicine, Royal Perth Hospital, Perth, Western Australia, Australia

${ }^{8}$ WA Centre for Health \& Ageing, University of Western Australia, Perth, Western Australia, Australia

${ }^{9}$ Cognitive Clinic, Royal Perth Hospital, Perth, Western Australia, Australia

${ }^{10}$ Academic Unit of Psychiatry of Old Age, University of Melbourne, Victoria, Victoria, Australia

${ }^{11}$ North Western Mental Health, Royal Melbourne Hospital, Parkville, Victoria, Australia

${ }^{12}$ Division of Psychiatry and WA Centre for Health and Ageing, University of Western Australia, Perth, Western Australia, Australia

${ }^{13}$ Synapse Neuropsychology, Perth, Western Australia, Australia

${ }^{14}$ Faculty of Health Sciences, Curtin University, Bentley, Western Australia, Australia

${ }^{15}$ School of Paediatrics and Child Health, Faculty of Health and Medical Science,

University of Western Australia, Crawley, Western Australia, Australia

${ }^{16}$ Department of Radiation Oncology, Sir Charles Gairdner Hospital, Nedlands,

Western Australia, Australia

${ }^{17}$ Duke-NUS Medical School, National University of Singapore, Singapore

${ }^{18}$ Institute for Research in Molecular Medicine, Universiti Sains Malaysia, Pulau

Pinang, Malaysia

${ }^{19}$ Curtin Medical School, Faculty of Health Sciences, Curtin University, Bentley, Western Australia, Australia

Twitter Christopher M Reid @profcmreid, Suzanne Robinson @Robinsonsuz and Ryusuke Takechi @Ryu_Takechi

Contributors VL, RC, RF, MB, SSD, RT and JM conceived the study concept. VL, $\mathrm{RC}, \mathrm{RF}, \mathrm{MB}, \mathrm{GW}, \mathrm{LF}, \mathrm{SSD}, \mathrm{EC}, \mathrm{RT}$ and JM designed the trial protocol. LF, CFO, PL, $\mathrm{NL}, \mathrm{CMR}, \mathrm{JKF}, \mathrm{SR}, \mathrm{MV}$ and BH commented on the methods and contributed to the development of the study. VL, EC and JM drafted the manuscript. All authors revised the manuscript and approved the final version.
Funding The PIA study is funded by the National Health and Medical Research Council Medical Research Future Fund for Neurological Disorders (MRF1201204), the Multiple Sclerosis Society of Western Australia (MSWA) and the McCusker Charitable Foundation.

Disclaimer Curtin University, the National Health and Medical Research Council and MSWA do not have any responsibility relating to study design; collection, management, analysis and interpretation of data; writing of the report; and the decision to submit the report for publication.

Competing interests None declared.

Patient consent for publication Not applicable.

Provenance and peer review Not commissioned; externally peer reviewed.

Supplemental material This content has been supplied by the author(s). It has not been vetted by BMJ Publishing Group Limited (BMJ) and may not have been peer-reviewed. Any opinions or recommendations discussed are solely those of the author(s) and are not endorsed by BMJ. BMJ disclaims all liability and responsibility arising from any reliance placed on the content. Where the content includes any translated material, BMJ does not warrant the accuracy and reliability of the translations (including but not limited to local regulations, clinical guidelines, terminology, drug names and drug dosages), and is not responsible for any error and/or omissions arising from translation and adaptation or otherwise.

Open access This is an open access article distributed in accordance with the Creative Commons Attribution Non Commercial (CC BY-NC 4.0) license, which permits others to distribute, remix, adapt, build upon this work non-commercially, and license their derivative works on different terms, provided the original work is properly cited, appropriate credit is given, any changes made indicated, and the use is non-commercial. See: http://creativecommons.org/licenses/by-nc/4.0/.

\section{ORCID iDs}

Virginie Lam http://orcid.org/0000-0001-8463-645X

Suzanne Robinson http://orcid.org/0000-0001-5703-6475

Emily Corti http://orcid.org/0000-0003-3130-6611

Ryusuke Takechi http://orcid.org/0000-0001-6359-3382

\section{REFERENCES}

1 van der Kall LM, Truong T, Burnham SC, et al. Association of $\beta$-amyloid level, clinical progression, and longitudinal cognitive change in normal older individuals. Neurology 2021;96:e662-70.

2 Cummings J, Aisen P, Lemere C, et al. Aducanumab produced a clinically meaningful benefit in association with amyloid lowering. Alzheimers Res Ther 2021;13:98.

3 Sevigny J, Chiao P, Bussière $T$, et al. The antibody aducanumab reduces A $\beta$ plaques in Alzheimer's disease. Nature 2016;537:50-6.

4 Zlokovic BV. Neurovascular pathways to neurodegeneration in Alzheimer's disease and other disorders. Nat Rev Neurosci 2011;12:723-38.

5 ladecola $\mathrm{C}$. The overlap between neurodegenerative and vascular factors in the pathogenesis of dementia. Acta Neuropathol 2010;120:287-96.

6 Tan C-C, Yu J-T, Wang H-F, et al. Efficacy and Safety of Donepezil, Galantamine, Rivastigmine, and Memantine for the Treatment of Alzheimer's Disease: A Systematic Review and Meta-Analysis. Journal of Alzheimer's Disease 2014;41:615-31.

7 Schindler SE, Bollinger JG, Ovod V, et al. High-precision plasma $\beta$-amyloid $42 / 40$ predicts current and future brain amyloidosis. Neurology 2019;93:e1647-59.

8 Nakamura A, Kaneko N, Villemagne VL, et al. High performance plasma amyloid- $\beta$ biomarkers for Alzheimer's disease. Nature 2018;554:249-54.

9 Matsubara E, Sekijima Y, Tokuda T, et al. Soluble Abeta homeostasis in $A D$ and Ds: impairment of anti-amyloidogenic protection by lipoproteins. Neurobiol Aging 2004;25:833-41.

10 Mamo JCL, Jian L, James AP, et al. Plasma lipoprotein beta-amyloid in subjects with Alzheimer's disease or mild cognitive impairment. Ann Clin Biochem 2008;45:395-403.

11 Lam V, Takechi R, Hackett MJ, et al. Synthesis of human amyloid restricted to liver results in an Alzheimer disease-like neurodegenerative phenotype. PLoS Biol 2021;19:e3001358.

12 Heel RC, Brogden RN, Speight TM, et al. Probucol: a review of its pharmacological properties and therapeutic use in patients with hypercholesterolaemia. Drugs 1978;15:409-28.

13 Parthasarathy S, Young SG, Witztum JL, et al. Probucol inhibits oxidative modification of low density lipoprotein. J Clin Invest 1986;77:641-4. 
14 Fruebis J, Gonzalez V, Silvestre M, et al. Effect of probucol treatment on gene expression of VCAM-1, MCP-1, and M-CSF in the aortic wall of $L D L$ receptor-deficient rabbits during early atherogenesis. Arterioscler Thromb Vasc Biol 1997;17:1289-302.

15 Pallebage-Gamarallage MM, Galloway S, Takechi R, et al. Probucol suppresses enterocytic accumulation of amyloid- $\beta$ induced by saturated fat and cholesterol feeding. Lipids 2012;47:27-34.

16 Mamo JC, Elsegood CL, Umeda Y, et al. Effect of probucol on plasma clearance and organ uptake of chylomicrons and VLDLs in normal and diabetic rats. Arterioscler Thromb 1993;13:231-9.

17 Takechi R, Galloway S, Pallebage-Gamarallage MM, et al. Probuco prevents blood-brain barrier dysfunction in wild-type mice induced by saturated fat or cholesterol feeding. Clin Exp Pharmacol Physiol 2013;40:45-52.

18 Mamo JC, Lam V, Brook E, et al. Probucol prevents blood-brain barrier dysfunction and cognitive decline in mice maintained on prodiabetic diet. Diab Vasc Dis Res 2019;16:87-97.

19 Chan A-W, Tetzlaff JM, Altman DG, et al. Spirit 2013 statement: defining standard protocol items for clinical trials. Ann Intern Med 2013;158:200.

20 Lekeu F, Van der Linden M, Chicherio C, et al. Brain correlates of performance in a free/cued recall task with semantic encoding in Alzheimer disease. Alzheimer Dis Assoc Disord 2003;17:35-45.

21 Berg L. Clinical dementia rating (CDR). Psychopharmacol Bull 1988;24:637-9.

22 Folstein MF, Folstein SE, McHugh PR. "Mini-mental state". A practical method for grading the cognitive state of patients for the clinician. J Psychiatr Res 1975;12:189-98.

23 Verma N, Beretvas SN, Pascual B, et al. New scoring methodology improves the sensitivity of the Alzheimer's disease assessment Scale-Cognitive subscale (ADAS-Cog) in clinical trials. Alzheimers Res Ther 2015;7:64.
24 Ritchie CW, Molinuevo JL, Truyen L, et al. Development of interventions for the secondary prevention of Alzheimer's dementia: the European prevention of Alzheimer's dementia (EPAD) project. Lancet Psychiatry 2016;3:179-86.

25 Wardlaw JM, Smith EE, Biessels GJ, et al. Neuroimaging standards for research into small vessel disease and its contribution to ageing and neurodegeneration. Lancet Neurol 2013;12:822-38.

26 Gregoire SM, Chaudhary UJ, Brown MM, et al. The microbleed anatomical rating scale (MARS): reliability of a tool to map brain microbleeds. Neurology 2009;73:1759-66.

27 Cordonnier C, Potter GM, Jackson CA, et al. Improving interrater agreement about brain microbleeds. Stroke 2009;40:94-9.

28 Pedrosa H, De Sa A, Guerreiro M, et al. Functional evaluation distinguishes $\mathrm{MCI}$ patients from healthy elderly people-the ADCS/ MCl/ADL scale. J Nutr Health Aging 2010;14:703-9.

29 Lovibond Set al. Manual for the depression anxiety stress scales. Australia: Sydney Psychology Foundation, 1995.

30 Sano M, Raman R, Emond J, et al. Adding delayed recall to the Alzheimer disease assessment scale is useful in studies of mild cognitive impairment but not Alzheimer disease. Alzheimer Dis Assoc Disord 2011;25:122-7.

31 Ledig C, Schuh A, Guerrero R, et al. Structural brain imaging in Alzheimer's disease and mild cognitive impairment: biomarker analysis and shared morphometry database. Sci Rep 2018;8:11258

32 Shimizu S, Hoyer PO, Hyvarinen A. A linear non-Gaussian acyclic model for causal discovery. J Mach Learn Res 2006;7:2003-30.

33 Chickering D. Optimal structure identification with greedy search. $J$ Mach Learn Res 2003;3:507-54.

34 Moher D, Hopewell S, Schulz KF, et al. Consort 2010 explanation and elaboration: updated guidelines for reporting parallel group randomised trials. Int J Surg 2012;10:28-55. 